\title{
Nanoscopic Charge Fluctuations in a Gallium Phosphide Waveguide Measured by Single Molecules
}

\author{
Alexey Shkarin, ${ }^{1, *}$ Dominik Rattenbacher $\odot,{ }^{1, *}$ Jan Renger $\odot,{ }^{1}$ Simon Hönl, ${ }^{2}$ Tobias Utikal®, ${ }^{1}$ \\ Paul Seidler, ${ }^{2}$ Stephan Götzinger $\odot,,^{3,1,4}$ and Vahid Sandoghdar $\oplus^{1,3}$ \\ ${ }^{1}$ Max Planck Institute for the Science of Light, D-91058 Erlangen, Germany \\ ${ }^{2}$ IBM Research Europe, Säumerstrasse 4, CH-8803 Rüschlikon, Switzerland \\ ${ }^{3}$ Department of Physics, Friedrich Alexander University Erlangen-Nuremberg, D-91058 Erlangen, Germany \\ ${ }^{4}$ Graduate School in Advanced Optical Technologies (SAOT), Friedrich Alexander University Erlangen-Nuremberg, \\ D-91052 Erlangen, Germany
}

(Received 27 November 2020; accepted 12 February 2021; published 2 April 2021)

\begin{abstract}
We present efficient evanescent coupling of single organic molecules to a gallium phosphide (GaP) subwavelength waveguide (nanoguide) decorated with microelectrodes. By monitoring their Stark shifts, we reveal that the coupled molecules experience fluctuating electric fields. We analyze the spectral dynamics of different molecules over a large range of optical powers in the nanoguide to show that these fluctuations are light-induced and local. A simple model is developed to explain our observations based on the optical activation of charges at an estimated mean density of $2.5 \times 10^{22} \mathrm{~m}^{-3}$ in the GaP nanostructure. Our work showcases the potential of organic molecules as nanoscopic sensors of the electric charge as well as the use of $\mathrm{GaP}$ nanostructures for integrated quantum photonics.
\end{abstract}

DOI: 10.1103/PhysRevLett.126.133602

One of the promising platforms for future quantum technologies is based on integrated nanophotonics, where a large number of quantum emitters and quantum states of light are efficiently interconnected via a labyrinth of subwavelength waveguides (nanoguides) and other nano-optical elements [1-5]. The optimal choice of materials for achieving this goal has not been settled yet, but semiconductors (e.g., GaAs) [6-8] and diamond [9] architectures have made significant progress. In most cases, quantum emitters have been situated inside the host material of the nanoguides, e.g., AlGaAs quantum dots in GaAs or color centers in diamond. To decouple the choices of ideal quantum emitters, appropriate guiding architectures, and other nano-optical elements from each other, there is a large effort toward hybrid solutions [2,3,10-15].

Guiding light on a chip is best realized in a material with high refractive index $n$. Single quantum emitters in the visible and near-infrared regimes have commonly been coupled to chip-based nanoguides made of silicon nitride [12,15-17], aluminum nitride [18], or titanium dioxide $[11,19]$, with $n \sim 2-2.5$. Semiconductors such as $\mathrm{Si}$ and GaAs offer considerably larger $n$ but at the expense of

Published by the American Physical Society under the terms of the Creative Commons Attribution 4.0 International license. Further distribution of this work must maintain attribution to the author(s) and the published article's title, journal citation, and DOI. Open access publication funded by the Max Planck Society. strong absorption for wavelengths smaller than $800 \mathrm{~nm}$. Gallium phosphide $(\mathrm{GaP})$ presents a very attractive alternative with $n \gtrsim 3$ and a cutoff wavelength below $600 \mathrm{~nm}$ [20-23], and indeed, recent efforts have established fabrication of high-quality nanophotonic chips from this material on $\mathrm{SiO}_{2}$ [24,25]. In this work, we evanescently couple single organic molecules to a $\mathrm{GaP}$ nanoguide and use them as local probes of the electric field. By examining the temporal properties of the molecular Stark shifts and their correlations for different molecules, we reveal lightinduced charge fluctuations in the $\mathrm{GaP}$ nanoguide with a spatial correlation length of less than $100 \mathrm{~nm}$.

Efficient coupling of molecules to a GaP nanoguide.The core of our experimental platform is a $50-\mu \mathrm{m}$-long GaP nanoguide fabricated on a $2-\mu$ m-thick $\mathrm{SiO}_{2}$ layer on a silicon substrate $[24,25]$. The nanoguide has a cross section of $100 \times 160 \mathrm{~nm}^{2}$, is terminated on both sides by grating couplers, and is decorated by sawtooth-shaped gold microelectrodes placed $2 \mu \mathrm{m}$ from the nanoguide [see Figs. 1(a) and 1(b)]. The nanoguide supports two propagating modes of orthogonal polarization states, which have the majority of their energy concentrated in the evanescent field [see Fig. 1(b)]. Fabrication details are presented in the Supplemental Material (SM) [26].

The organic molecule chosen for the current study is dibenzoterrylene (DBT) doped at a concentration of $2 \times 10^{-4} \mathrm{~mol} / \mathrm{mol}$ into para-dichlorobenzene ( $p$-DCB). The doped $p$-DCB crystal covers the sample chip and is confined in a microchannel as described in previous publications $[11,19,30]$, leading to a random distribution 

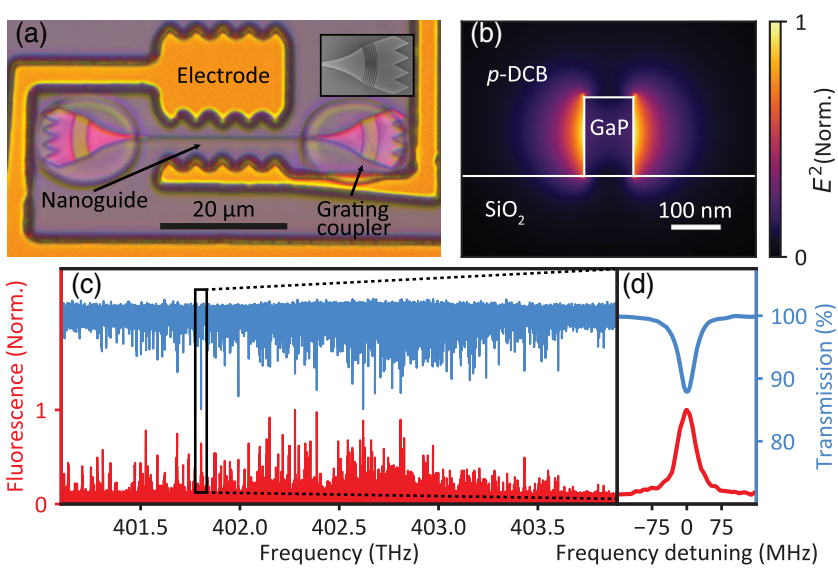

FIG. 1. (a) An optical image of a nanoguide interfaced with two electrodes. The grating couplers on both sides of the nanoguide are covered by a polymer to shield them from irregularities in the $p$-DCB crystal [19]. The inset shows a scanning electron microscope image of a grating coupler before covering it with the polymer. (b) Simulated intensity profile of the TE mode in the nanoguide. (c) Extinction (blue) and fluorescence (red) spectra of hundreds of molecules. (d) Extinction and fluorescence spectra of one single molecule.

of the DBT molecules around the nanoguides. At a temperature below $4 \mathrm{~K}$, DBT molecules possess strong zero-phonon lines with a lifetime-limited linewidth of $30 \mathrm{MHz}$ at a vacuum transition wavelength of $745 \mathrm{~nm}$ (frequency of $402 \mathrm{THz}$ ) [31,32]. Owing to variations in their nanoscopic crystal environment [30], the resonance frequencies of individual molecules span a range of about $4 \mathrm{~nm}(2 \mathrm{THz})$.

The details of the optical setup are discussed in the SM [26]. The transverse electric (TE) mode of the nanoguide [see Fig. 1(b)] is excited by continuous-wave Ti:sapphire laser beams (linewidth $<1 \mathrm{MHz}$ ), and the out-coupled radiation is detected on an avalanche photodiode. The blue curve in Fig. 1(c) displays the extinction spectra of several hundred molecules measured in transmission, while the red spectrum reports on the corresponding fluorescence via the vibrational levels of the ground state and phonon wings. In Fig. 1(d), we plot a close-up for a single-molecule extinction dip of $13 \%$, comparable with the coupling efficiency achieved for illumination in a diffraction-limited focus spot [33].

Monitoring the local electric field.-The molecule DBT has inversion symmetry and is thus expected to undergo a quadratic Stark shift. Figure 2(a) illustrates the parabolic spectra of three nanoguide-coupled molecules as a function of the voltage applied to the nearby electrodes. In some cases, local strain in the matrix leads to the addition of a linear Stark effect manifested by an offset in the turning points of the parabolas [34,35]. A more noteworthy observation in these spectra is, however, that the resonances appear broader and less stable at larger slopes of the Stark tuning profile [see, e.g., the spectra across the dashed line in
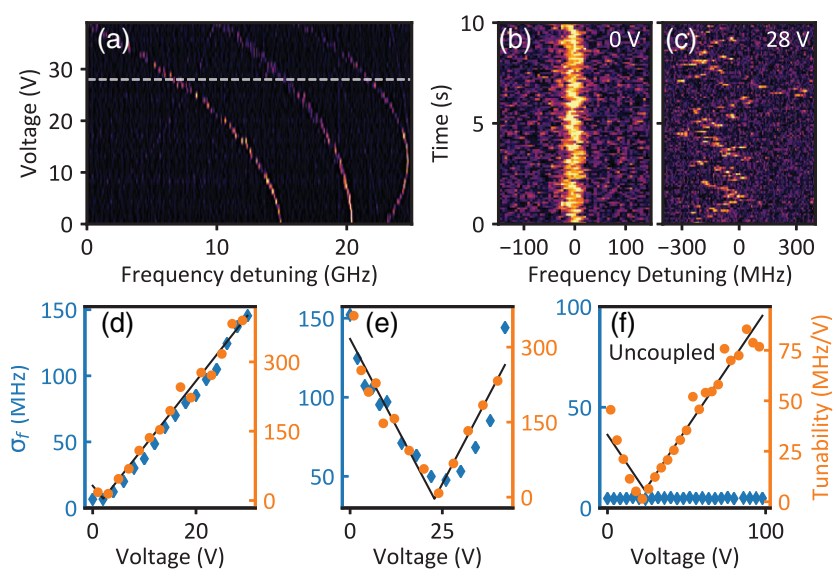

FIG. 2. (a) Fluorescence signals of three molecules as a function of laser frequency detuning and applied external voltage. The dashed line points to broad instable resonances at high voltages. (b),(c) Temporal spectral dynamics of a single nanoguide-coupled molecule (M1) studied under two different applied $\mathrm{dc}$ voltages of 0 and $28 \mathrm{~V}$. Individual spectra were recorded at the rate of $10 \mathrm{scans} / \mathrm{s}$. The color scheme in (a)-(c) is chosen to highlight the molecular resonance frequency. (d),(e) Frequency fluctuations $\sigma_{f}$ (blue, diamond) and absolute tunability $\partial f / \partial V$ (orange, circle) as a function of applied voltage for two nanoguide-coupled molecules. The molecule in (d) was also M1. (f) Same as (d) and (e) but for a molecule far from the nanoguide. Black lines are linear fits to the absolute value of tunability.

Fig. 2(a)]. We point out that this effect is consistently observed for all nanoguide-coupled molecules on all studied GaP samples. On the contrary, we have verified that molecules at about $10 \mu \mathrm{m}$ away from the nanoguide are highly stable and have a lifetime-limited linewidth regardless of the applied Stark shift. For the latter measurements, we shined a focused laser beam normal to the plane of the chip.

We, thus, attribute the origin of the spectral instabilities for nanoguide-coupled molecules to electric field fluctuations originating from the GaP nanostructure. As we show in the following, the effect at hand is very different from previous reports of electric field-induced spectral instability and broadening of organic molecules, which were caused by two-level tunneling systems [36-41] or electromechanical oscillations [42] in the host organic matrix.

To investigate the temporal dynamics of the spectral instabilities more quantitatively, we increased the frequency scan rate from $400 \mathrm{MHz} / \mathrm{s}$ in Fig. 2(a) to $10 \mathrm{GHz} / \mathrm{s}$. This was sufficient to arrive at lifetime-limited Lorentzian resonances in individual frequency sweeps and, thus, resolve the wandering of their center frequencies over time. To characterize Stark spectra such as those shown in Fig. 2(a), we recorded a large number of individual scans. In this manner, we obtained a robust mean value $(f)$ for the molecular resonance frequency and root mean square (rms) frequency fluctuations $\left(\sigma_{f}\right)$ at a given electrode voltage $(V)$. 
By repeating this procedure for many applied voltages, we established the frequency tunability defined as $\partial f / \partial V$. Figures 2(b) and 2(c) display examples of spectral trajectories of a molecule, which we name M1, at two applied voltages.

Figures 2(d)-2(f) show $\sigma_{f}$ and the tunability for three exemplary molecules. For M1, $\partial f / \partial V$ (orange) increases linearly with $V$, as shown in Fig. 2(d), implying a quadratic Stark behavior. Interestingly, $\sigma_{f}$ (blue) also grows proportionally with $\partial f / \partial V$. Figure 2(e) presents the case of a second nanoguide-coupled molecule that behaves similarly although its turning point $(\partial f / \partial V=0)$ is shifted to a voltage of about $25 \mathrm{~V}$. As a control experiment, in Fig. 2(f) we report on a molecule far away from the nanoguide. It is evident that, in this case, there is no correlation between $\partial f / \partial V$ and $\sigma_{f}$, which stays at $4 \mathrm{MHz}$, given by the fit error.

Assuming that the field fluctuations originate from the nanoguide, we express the total Stark shift experienced by the molecule as $\delta f \propto\left|\mathbf{E}_{\mathrm{cr}}+\mathbf{E}_{\mathrm{el}}+\mathbf{E}_{\mathrm{ng}}\right|^{2}$, where $\mathbf{E}_{\mathrm{cr}}, \mathbf{E}_{\mathrm{el}}$, and $\mathbf{E}_{\mathrm{ng}}$ denote the fields created by the residual strain in the crystal, the electrodes, and the nanoguide, respectively. A cross term in this expression leads to the amplification of a small fluctuating $\mathbf{E}_{\mathrm{ng}}$ by a large constant $\left(\mathbf{E}_{\mathrm{cr}}+\mathbf{E}_{\mathrm{el}}\right)$. Here, $\mathbf{E}_{\text {ng }}$ causes $\sigma_{f}$ while $\left(\mathbf{E}_{\mathrm{cr}}+\mathbf{E}_{\mathrm{el}}\right)$ dictates $\partial f / \partial V$ such that $\sigma_{f}$ becomes proportional to $\partial f / \partial V$, as observed in our measurements. The measured data in Figs. 2(d) and 2(e) and our knowledge of the electrode and nanoguide geometries let us deduce the rms nanoguide field fluctuations to be around $40 \mathrm{kV} / \mathrm{m}$ at the position of the molecule [26]. This corresponds to the field generated by a single electron at a distance of $100 \mathrm{~nm}$, which is comparable to the typical separation of the evanescently coupled nanoguidemolecule system.

Temporal field correlations. - The ability to scan faster than typical electric field fluctuations allows us to determine the correlation function $C_{j k}(\Delta t)$ between the frequency fluctuations of molecules $j$ and $k$. Figure 3(a) plots the autocorrelation function $C_{11}$ computed for molecule M1 from 12000 individual sweeps, 100 of which are displayed in Fig. 3(b). By fitting an exponential function to $C_{11}$, we extract the autocorrelation time $\tau=1.0 \mathrm{~s}$ at an optical power $(P)$ of $3 \times 10^{7}$ photons/s in the nanoguide (see SM for details [26]).

Next, we studied the dependence of $\tau$ and $\sigma_{f}$ on $P$. Figures 3(c) and 3(d) display the outcome of measurements for one molecule, indicating an inverse proportionality relation between $\tau$ and $P$ over 4 orders of magnitude. This robust dependency clearly demonstrates that the fluctuations are photoinduced. We checked that the observed phenomenon is not caused by direct interaction of light with the molecule. To do this, we performed measurements, where up to $90 \%$ of the optical power inside the nanoguide was provided by a second laser beam detuned by $2 \mathrm{THz}$ from the molecule and verified that the fluctuation rate $(1 / \tau)$ scaled with the total laser power in the nanoguide.

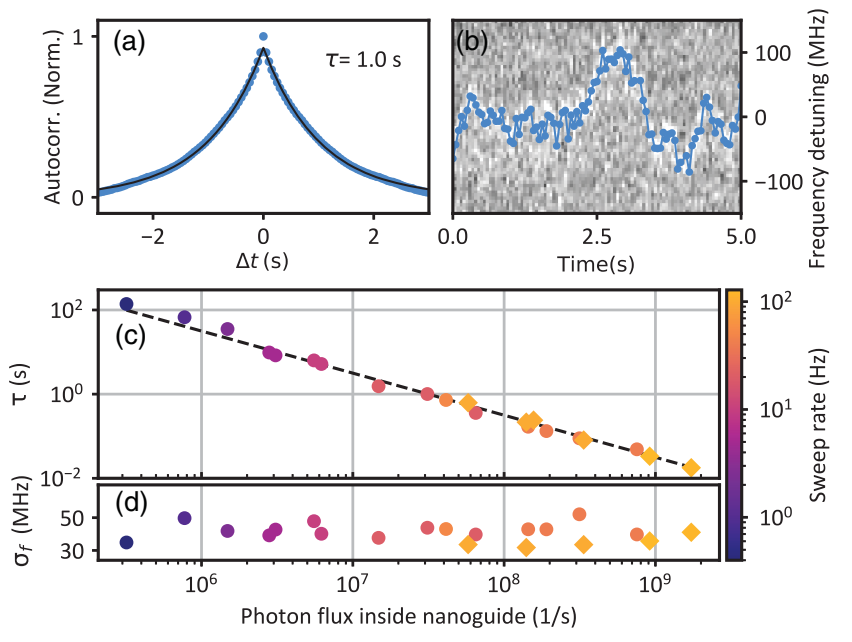

FIG. 3. (a) Autocorrelation function of the frequency trace of molecule M1 normalized to $C_{11}(0)$, measured at $10 \mathrm{~V}$ and an optical power of $3 \times 10^{7}$ photons/s. Black line shows an exponential fit function, yielding an autocorrelation time of $\tau=1.0 \mathrm{~s}$. (b) Nanoguide transmission for repeated scans of the laser frequency. The blue symbols highlight the fitted resonance frequencies for each sweep. (c) Dependence of $\tau$ on the optical power $(P)$ in the nanoguide. See SM for details on the power calibration [26]. Dots (diamonds) denote results obtained through extinction (fluorescence) measurements. The color coding indicates the sweep rate used to record the frequency traces. The straight line shows a fit to $\tau \propto P^{-1}$. (d) Magnitude of the center frequency fluctuations.

Furthermore, we found that $\sigma_{f}$ remains independent of $P$ within our measurement precision [see Fig. 3(d)]. This indicates that while the external illumination power dictates the spectral noise dynamics, it does not influence its amplitude.

Spatial field correlations.-We now investigate the spatial properties of the observed electric field noise by simultaneous measurement of several molecules. Here, we coupled two laser beams into the nanoguide, the amplitudes and frequencies of which were controlled independently. By gating the laser amplitudes in a synchronous manner, we ensured that only one laser was on at a given time, resulting in alternating sweeps of two separate frequency regions. This yielded near-simultaneous measurements of two molecules (for details, see Sec. II of the SM [26]). In Fig. 4, we present an example, where the frequency fluctuations of three molecules [M1, M2, and M3; see Fig. 4(a)] were examined. The frequency variations and the cross-correlation functions $C_{12}, C_{23}$ displayed in Figs. 4(b) and 4(c), respectively, reveal that the spectral fluctuations in M1 and M2 are correlated, but M3 behaves independently. Considering the error in extracting resonance frequencies, we infer almost perfect correlation between M1 and M2 (see SM [26]). By determining the positions of each molecule through localization microscopy [26], we revealed that, indeed, M1 and M2 were separated by only 


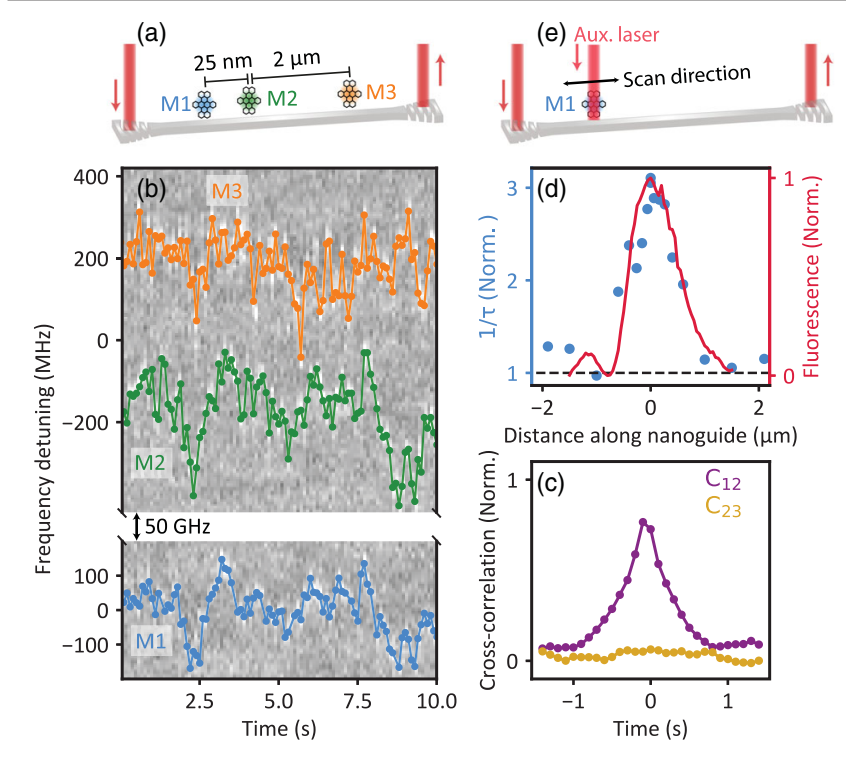

FIG. 4. (a) Sketch of the arrangement of molecules labeled M1, M2, and M3 along the nanoguide. (b) Examples of transmission spectra obtained simultaneously at two frequency intervals separated by about $50 \mathrm{GHz}$. Colored lines indicate the evolution of the resonance frequencies. (c) Cross-correlation functions of M1-M2 $\left(C_{12}\right.$, magenta) and M2-M3 $\left(C_{23}\right.$, mustard). (d) Frequency fluctuation rate $1 / \tau$ (blue) as a function of the displacement of an auxiliary focused laser beam along the nanoguide over a molecule; see (e). The rate is normalized to that in the absence of the auxiliary excitation. The red curve shows the intensity profile of the excitation laser's focus spot mapped by the fluorescence of the molecule. (e) Sketch of the arrangement when a second laser beam is shone in the direction normal to the plane of the chip and its focus spot is scanned along the nanoguide.

$26 \pm 5 \mathrm{~nm}$, while M3 was about $2 \mu \mathrm{m}$ away from them. In another example (see SM [26]), we show that the peak spectral cross-correlation of a different pair of molecules separated by a larger distance of about $80 \mathrm{~nm}$ drops to $\sim 30 \%$, verifying that the field fluctuations are very local.

To provide further evidence for the local character of the field fluctuations, we also conducted single-molecule measurements under illumination by an auxiliary laser beam that was frequency detuned by $6 \mathrm{GHz}$. Here, we scanned the focal spot of this second beam along the nanoguide across the molecule position [see Figs. 4(d) and $4(\mathrm{e})]$ and recorded changes in $1 / \tau$. The blue symbols in Fig. 4(d) confirm that $1 / \tau$ follows the intensity profile of the auxiliary light beam shown by the red curve (full width at half maximum $1 \mu \mathrm{m}$ ).

Theoretical modeling.-Regardless of the details of the process at work, electric field fluctuations generated by the nanoguide can be attributed to charge fluctuations. Redistribution of charges in semiconductors can be caused by many effects such as trapped or wandering charges [43-48], impurities [49], or ligand rearrangements [50,51] and have also been shown to be driven by light with energy

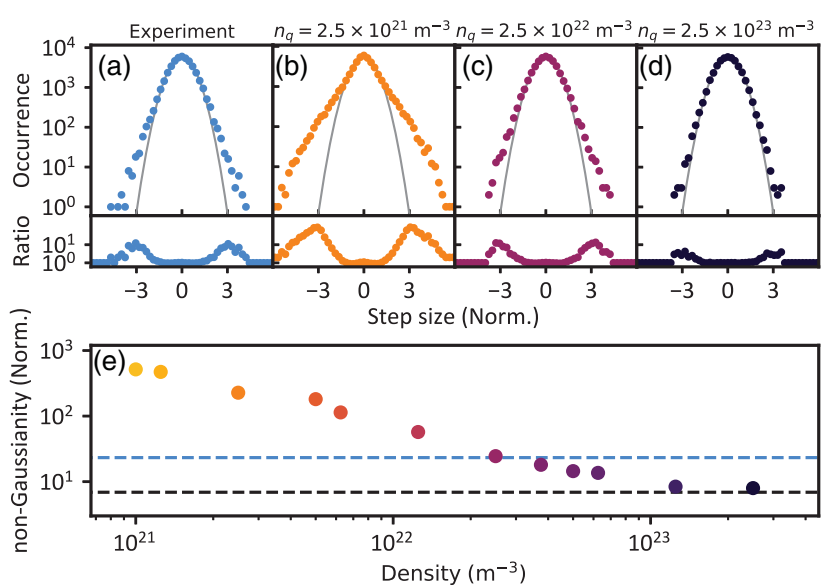

FIG. 5. Upper plots: Histograms of the step-size distributions for the experimental data (a) and three synthetic datasets at different densities $n_{q}$ (b)-(d). Gray curves denote fits to a Gaussian distribution. Lower plots: Ratio of the occurrences to their corresponding Gaussian functions after an offset correction (see SM [26]). (e) The symbols show the dependence of the nonGaussianity parameter $\eta$ (see text) on the simulated charge density used. The blue horizontal line corresponds to the experimental data, and the black line signifies the baseline of our analysis caused by fit errors [26].

below the band gap [52]. We consider a simple model in which electric field fluctuations result from the rearrangement of randomly distributed point charges with density $n_{q}$ in the GaP nanoguide. We assume that each charge stays in the vicinity of its original position, but it experiences an average displacement $d$ upon scattering one photon. In other words, while the field fluctuation rate scales with the optical power $P, n_{q}$ and $d$ remain independent of it. It can then be shown that $\sigma_{f} \propto d \sqrt{n_{q}}$ (see SM [26]). Furthermore, the randomness of the jumps and the quadratic falloff of the Coulomb field lead to a short correlation length, approximately equal to the molecule-waveguide distance (see SM [26]).

In the limit of large $n_{q}$, one expects a Gaussian statistic for the frequency steps. The upper panel of Fig. 5(a) shows that the histogram of the measured data clearly deviates from a Gaussian distribution shown in gray. To compare the statistical properties of the frequency fluctuations with the predictions of our model, we simulated time traces of the molecular resonance frequencies for different $n_{q}$ values and used the same analysis procedure applied to the experimental data to generate a histogram of the frequency steps (see SM [26] for details). The upper panel of Figs. 5(b)-5(d) displays the outcome for three synthetic datasets. It turns out that at the lowest considered density, corresponding to the effect of only $\sim 5$ charges, the molecular resonance experiences jumplike frequency shifts, causing a non-Gaussian frequency step-size distribution.

To quantify the deviations from a Gaussian distribution, we consider the ratio of the occurrence to the corresponding Gaussian fit distribution as plotted in the lower panel of 
Figs. 5(a)-5(d). Next, as presented in Fig. 5(e), we introduce a measure of non-Gaussianity $(\eta)$, defined as the area under this curve above one [26]. The experimentally measured $\eta$ marked by the blue line corresponds to a density of charges around $n_{q}=2.5 \times 10^{22} \mathrm{~m}^{-3}$, or equivalently to about 1 charge per $(35 \mathrm{~nm})^{3}$. Although this analysis only establishes an estimate, the result is in agreement with the typical density of defects, impurities, or charge traps seen in such systems [49] and implies that an average of 50 charges govern the frequency fluctuations of our molecules. Given this density and the experimentally measured magnitude of the frequency fluctuations, we can estimate $d \approx 20 \mathrm{~nm}$ [26]. We note that although we have considered volume charges in the discussion of Fig. 5, we have also explored the effect of fluctuating surface charges [53-56] in the SM [26]. We find that owing to the small width of the nanoguide, surface and volume charges lead to similar results. Further experiments could vary the cross-sectional dimensions of the nanoguide or apply a passivation layer to its surface in order to investigate the origin of the charge fluctuations.

Conclusions. - We have used single molecules as nanometer-sized probes for investigating the spatiotemporal behavior of a low number of charges activated in GaP nanoguides. The small size, excellent spectral properties, large achievable concentrations, and the inhomogeneous distribution of their resonance frequencies make organic molecules a promising tool for ultrasensitive characterization of nanoscopic charge dynamics in a range of systems such as single electron transistors, quantum dots, or superconductors [57-62]. Our findings also advance the use of $\mathrm{GaP}$ as a platform for integrated quantum photonics $[1,2,4,23]$. The observed light-induced field fluctuations are small and slow enough to be tolerated or eliminated by more sophisticated fabrication schemes $[48,55]$. However, even in their current form, the estimated density of charges and their light absorption probability signified by the slope of Fig. 3(c) point to a loss coefficient of about $0.5 \mathrm{~dB} / \mathrm{cm}$ [26], which would permit ring resonator quality factors in the order of $10^{6}$ at a wavelength of $\sim 740 \mathrm{~nm}$.

We acknowledge financial support by the Max Planck Society as well as the RouTe Project (13N14839) through the Federal Ministry of Education and Research (BMBF) and the European Union's Horizon 2020 Program for Research and Innovation under Grants No. 722923 (Marie Curie H2020-ETN OMT) and No. 732894 (FET Proactive HOT). V.S. thanks Klaus Ensslin for helpful discussions. A.S. acknowledges support through an Alexander von Humboldt fellowship.

*These authors contributed equally to this work.

[1] J. Wang, F. Sciarrino, A. Laing, and M. G. Thompson, Integrated photonic quantum technologies, Nat. Photonics 14, 273 (2020).
[2] J.-H. Kim, S. Aghaeimeibodi, J. Carolan, D. Englund, and E. Waks, Hybrid integration methods for on-chip quantum photonics, Optica 7, 291 (2020).

[3] A. W. Elshaari, W. Pernice, K. Srinivasan, O. Benson, and V. Zwiller, Hybrid integrated quantum photonic circuits, Nat. Photonics 14, 285 (2020).

[4] V. Sandoghdar, Nano-optics in $2020 \pm 20$, Nano Lett. 20, 4721 (2020).

[5] P. Türschmann, H. L. Jeannic, S. F. Simonsen, H. R. Haakh, S. Götzinger, V. Sandoghdar, P. Lodahl, and N. Rotenberg, Coherent nonlinear optics of quantum emitters in nanophotonic waveguides, Nanophotonics 8, 1641 (2019).

[6] H. Thyrrestrup, G. Kiršanskè, H. Le Jeannic, T. Pregnolato, L. Zhai, L. Raahauge, L. Midolo, N. Rotenberg, A. Javadi, R. Schott, A. D. Wieck, A. Ludwig, M. C. Löbl, I. Söllner, R. J. Warburton, and P. Lodahl, Quantum optics with nearlifetime-limited quantum-dot transitions in a nanophotonic waveguide, Nano Lett. 18, 1801 (2018).

[7] D. Hallett, A. P. Foster, D. L. Hurst, B. Royall, P. Kok, E. Clarke, I. E. Itskevich, A. M. Fox, M. S. Skolnick, and L. R. Wilson, Electrical control of nonlinear quantum optics in a nano-photonic waveguide, Optica 5, 644 (2018).

[8] J. Q. Grim, A. S. Bracker, M. Zalalutdinov, S. G. Carter, A. C. Kozen, M. Kim, C. S. Kim, J. T. Mlack, M. Yakes, B. Lee, and D. Gammon, Scalable in operando strain tuning in nanophotonic waveguides enabling three-quantum-dot superradiance, Nat. Mater. 18, 963 (2019).

[9] M. Atatüre, D. Englund, N. Vamivakas, S.-Y. Lee, and J. Wrachtrup, Material platforms for spin-based photonic quantum technologies, Nat. Rev. Mater. 3, 38 (2018).

[10] A. V. Akimov, A. Mukherjee, C. L. Yu, D. E. Chang, A. S. Zibrov, P. R. Hemmer, H. Park, and M. D. Lukin, Generation of single optical plasmons in metallic nanowires coupled to quantum dots, Nature (London) 450, 402 (2007)

[11] P. Türschmann, N. Rotenberg, J. Renger, I. Harder, O. Lohse, T. Utikal, S. Götzinger, and V. Sandoghdar, Chipbased all-optical control of single molecules coherently coupled to a nanoguide, Nano Lett. 17, 4941 (2017).

[12] P. Lombardi, A. P. Ovvyan, S. Pazzagli, G. Mazzamuto, G. Kewes, O. Neitzke, N. Gruhler, O. Benson, W. H. P. Pernice, F. S. Cataliotti, and C. Toninelli, Photostable molecules on chip: Integrated sources of nonclassical light, ACS Photonics 5, 126 (2018).

[13] S. Grandi, M. P. Nielsen, J. Cambiasso, S. Boissier, K. D. Major, C. Reardon, T. F. Krauss, R. F. Oulton, E. A. Hinds, and A.S. Clark, Hybrid plasmonic waveguide coupling of photons from a single molecule, APL Photonics 4, 086101 (2019)

[14] C. U. Hail, C. Höller, K. Matsuzaki, P. Rohner, J. Renger, V. Sandoghdar, D. Poulikakos, and H. Eghlidi, Nanoprinting organic molecules at the quantum level, Nat. Commun. 10, 1880 (2019).

[15] J. E. Fröch, S. Kim, N. Mendelson, M. Kianinia, M. Toth, and I. Aharonovich, Coupling hexagonal boron nitride quantum emitters to photonic crystal cavities, ACS Nano 14, 7085 (2020).

[16] N. H. Wan, T.-J. Lu, K. C. Chen, M.P. Walsh, M.E. Trusheim, L. De Santis, E. A. Bersin, I. B. Harris, S. L. Mouradian, I. R. Christen, E. S. Bielejec, and D. Englund, 
Large-scale integration of artificial atoms in hybrid photonic circuits, Nature (London) 583, 226 (2020).

[17] S. Boissier, R. C. Schofield, L. Jin, A. Ovvyan, S. Nur, F. H. L. Koppens, C. Toninelli, W. H. P. Pernice, K. D. Major, E. A. Hinds, and A. S. Clark, Coherent characterisation of a single molecule in a photonic black box, Nat. Commun. 12, 706 (2021).

[18] T.-J. Lu, B. Lienhard, K.-Y. Jeong, H. Moon, A. Iranmanesh, G. Grosso, and D. Englund, Bright high-purity quantum emitters in aluminum nitride integrated photonics, ACS Photonics 7, 2650 (2020).

[19] D. Rattenbacher, A. Shkarin, J. Renger, T. Utikal, S. Götzinger, and V. Sandoghdar, Coherent coupling of single molecules to on-chip ring resonators, New J. Phys. 21, 062002 (2019).

[20] P. E. Barclay, K.-M. C. Fu, C. Santori, and R. G. Beausoleil, Chip-based microcavities coupled to nitrogen-vacancy centers in single crystal diamond, Appl. Phys. Lett. 95, 191115 (2009).

[21] J. Wolters, A. W. Schell, G. Kewes, N. Nüsse, M. Schoengen, H. Döscher, T. Hannappel, B. Löchel, M. Barth, and O. Benson, Enhancement of the zero phonon line emission from a single nitrogen vacancy center in a nanodiamond via coupling to a photonic crystal cavity, Appl. Phys. Lett. 97, 141108 (2010).

[22] M. Gould, E. R. Schmidgall, S. Dadgostar, F. Hatami, and K.-M. C. Fu, Efficient Extraction of Zero-Phonon-Line Photons from Single Nitrogen-Vacancy Centers in an Integrated GaP-on-Diamond Platform, Phys. Rev. Applied 6, 011001(R) (2016).

[23] D. J. Wilson, K. Schneider, S. Hönl, M. Anderson, Y. Baumgartner, L. Czornomaz, T. J. Kippenberg, and P. Seidler, Integrated gallium phosphide nonlinear photonics, Nat. Photonics 14, 57 (2020).

[24] S. Hönl, H. Hahn, Y. Baumgartner, L. Czornomaz, and P. Seidler, Highly selective dry etching of $\mathrm{GaP}$ in the presence of $\mathrm{Al}_{x} \mathrm{Ga}_{1-x}$ with a $\mathrm{SiCl}_{4} / \mathrm{SF}_{6}$ plasma, J. Phys. D 51, 185203 (2018).

[25] K. Schneider, P. Welter, Y. Baumgartner, H. Hahn, L. Czornomaz, and P. Seidler, Gallium phosphide-on-silicon dioxide photonic devices, J. Lightwave Technol. 36, 2994 (2018).

[26] See Supplemental Material at http://link.aps.org/ supplemental/10.1103/PhysRevLett.126.133602 for more details about the sample fabrication, the experimental setup, single emitter coupling strength simulations, the analysis of the autocorrelation function, the power calibration used for Fig. 3, the localization measurements, another correlated molecule pair, and the model, which includes Refs. [27-29].

[27] N. Rotenberg, P. Türschmann, H. R. Haakh, D. MartinCano, S. Götzinger, and V. Sandoghdar, Small slot waveguide rings for on-chip quantum optical circuits, Opt. Express 25, 5397 (2017).

[28] N. R. Verhart, G. Lepert, A. L. Billing, J. Hwang, and E. A. Hinds, Single dipole evanescently coupled to a multimode waveguide, Opt. Express 22, 19633 (2014).

[29] C. J. Foot, Atomic Physics (Oxford University Press, Oxford, England, 2005).

[30] B. Gmeiner, A. Maser, T. Utikal, S. Götzinger, and V. Sandoghdar, Spectroscopy and microscopy of single molecules in nanoscopic channels: spectral behavior vs. confinement depth, Phys. Chem. Chem. Phys. 18, 19588 (2016).

[31] A. A. L. Nicolet, P. Bordat, C. Hofmann, M. A. Kol'chenko, B. Kozankiewicz, R. Brown, and M. Orrit, Single dibenzoterrylene molecules in an anthracene crystal: Main insertion sites, ChemPhysChem 8, 1929 (2007).

[32] N. R. Verhart, M. Müller, and M. Orrit, Spectroscopy of Single Dibenzoterrylene Molecules in para-Dichlorobenzene, ChemPhysChem 17, 1524 (2016).

[33] G. Wrigge, I. Gerhardt, J. Hwang, G. Zumofen, and V. Sandoghdar, Efficient coupling of photons to a single molecule and the observation of its resonance fluorescence, Nat. Phys. 4, 60 (2008).

[34] M. Orrit, J. Bernard, A. Zumbusch, and R. Personov, Stark effect on single molecules in a polymer matrix, Chem. Phys. Lett. 196, 595 (1992).

[35] A. Moradi, Z. Ristanović, M. Orrit, I. Deperasińska, and B. Kozankiewicz, Matrix-induced linear stark effect of single dibenzoterrylene molecules in 2,3-dibromonaphthalene crystal, ChemPhysChem 20, 55 (2019).

[36] W. A. Phillips, Tunneling states in amorphous solids, J. Low Temp. Phys. 7, 351 (1972).

[37] A. Heuer and R. J. Silbey, Microscopic Description of Tunneling Systems in a Structural Model Glass, Phys. Rev. Lett. 70, 3911 (1993).

[38] H. Maier, R. Wunderlich, D. Haarer, B. M. Kharlamov, and S. G. Kulikov, Optical Detection of Electric Two Level System Dipoles in a Polymeric Glass, Phys. Rev. Lett. 74, 5252 (1995).

[39] J.-M. Segura, G. Zumofen, A. Renn, B. Hecht, and U. Wild, Tip-induced spectral dynamics of single molecules, Chem. Phys. Lett. 340, 77 (2001).

[40] M. Bauer and L. Kador, Electric-field effects of two-level systems observed with single-molecule spectroscopy, J. Chem. Phys. 118, 9069 (2003).

[41] I. Gerhardt, G. Wrigge, and V. Sandoghdar, Control and imaging of single-molecule spectral dynamics using a nanoelectrode, Mol. Phys. 107, 1975 (2009).

[42] Y. Tian, P. Navarro, and M. Orrit, Single Molecule as a Local Acoustic Detector for Mechanical Oscillators, Phys. Rev. Lett. 113, 135505 (2014).

[43] J. Müller, J. M. Lupton, A. L. Rogach, J. Feldmann, D. V. Talapin, and $\mathrm{H}$. Weller, Monitoring surface charge migration in the spectral dynamics of single $\mathrm{CdSe} / \mathrm{CdS}$ nanodot/ nanorod heterostructures, Phys. Rev. B 72, 205339 (2005).

[44] G. Sallen, A. Tribu, T. Aichele, R. André, L. Besombes, C. Bougerol, M. Richard, S. Tatarenko, K. Kheng, and J. P. Poizat, Subnanosecond spectral diffusion of a single quantum dot in a nanowire, Phys. Rev. B 84, 041405(R) (2011).

[45] R. Bardoux, T. Guillet, P. Lefebvre, T. Taliercio, T. Bretagnon, S. Rousset, B. Gil, and F. Semond, Photoluminescence of single GaN/AIN hexagonal quantum dots on Si(111): Spectral diffusion effects, Phys. Rev. B 74, 195319 (2006).

[46] J. Wolters, N. Sadzak, A. W. Schell, T. Schröder, and O. Benson, Measurement of the Ultrafast Spectral Diffusion of the Optical Transition of Nitrogen Vacancy Centers in Nano-Size Diamond Using Correlation Interferometry, Phys. Rev. Lett. 110, 027401 (2013). 
[47] A. Thoma, P. Schnauber, M. Gschrey, M. Seifried, J. Wolters, J.-H. Schulze, A. Strittmatter, S. Rodt, A. Carmele, A. Knorr, T. Heindel, and S. Reitzenstein, Exploring Dephasing of a Solid-State Quantum Emitter via Timeand Temperature-Dependent Hong-Ou-Mandel Experiments, Phys. Rev. Lett. 116, 033601 (2016).

[48] J. Liu, K. Konthasinghe, M. Davanço, J. Lawall, V. Anant, V. Verma, R. Mirin, S. W. Nam, J. D. Song, B. Ma, Z. S. Chen, H. Q. Ni, Z.C. Niu, and K. Srinivasan, Single Self-Assembled InAs/GaAs Quantum Dots in Photonic Nanostructures: The Role of Nanofabrication, Phys. Rev. Applied 9, 064019 (2018).

[49] M. Hauck, F. Seilmeier, S. E. Beavan, A. Badolato, P. M. Petroff, and A. Högele, Locating environmental charge impurities with confluent laser spectroscopy of multiple quantum dots, Phys. Rev. B 90, 235306 (2014).

[50] M. J. Fernée, T. Plakhotnik, Y. Louyer, B. N. Littleton, C. Potzner, P. Tamarat, P. Mulvaney, and B. Lounis, Spontaneous spectral diffusion in CdSe quantum dots, J. Phys. Chem. Lett. 3, 1716 (2012).

[51] A. P. Beyler, L. F. Marshall, J. Cui, X. Brokmann, and M. G. Bawendi, Direct Observation of Rapid Discrete Spectral Dynamics in Single Colloidal CdSe-CdS Core-Shell Quantum Dots, Phys. Rev. Lett. 111, 177401 (2013).

[52] J. J. W. Tomm, Spectroscopic Analysis of Optoelectronic Semiconductors (Springer International Publishing, Basel, Switzerland, 2016).

[53] M. Borselli, T. J. Johnson, and O. Painter, Beyond the Rayleigh scattering limit in high- $Q$ silicon microdisks: theory and experiment, Opt. Express 13, 1515 (2005).

[54] D. Parrain, C. Baker, G. Wang, B. Guha, E. G. Santos, A. Lemaitre, P. Senellart, G. Leo, S. Ducci, and I. Favero,
Origin of optical losses in gallium arsenide disk whispering gallery resonators, Opt. Express 23, 19656 (2015).

[55] B. Guha, F. Marsault, F. Cadiz, L. Morgenroth, V. Ulin, V. Berkovitz, A. Lemaître, C. Gomez, A. Amo, S. Combrié, B. Gérard, G. Leo, and I. Favero, Surface-enhanced gallium arsenide photonic resonator with quality factor of $6 \times 10^{6}$, Optica 4, 218 (2017).

[56] I. Rousseau, G. Callsen, G. Jacopin, J.-F. Carlin, R. Butté, and N. Grandjean, Optical absorption and oxygen passivation of surface states in III-nitride photonic devices, J. Appl. Phys. 123, 113103 (2018).

[57] M. Rezai, J. Wrachtrup, and I. Gerhardt, Coherence Properties of Molecular Single Photons for Quantum Networks, Phys. Rev. X 8, 031026 (2018).

[58] J. M. Caruge and M. Orrit, Probing local currents in semiconductors with single molecules, Phys. Rev. B 64, 205202 (2001).

[59] M. Fauré, B. Lounis, and A. I. Buzdin, Single-molecule spectroscopy as a possible tool to study the electric field in superconductors, Europhys. Lett. 77, 17005 (2007).

[60] A. N. Vamivakas, Y. Zhao, S. Fält, A. Badolato, J. M. Taylor, and M. Atatüre, Nanoscale Optical Electrometer, Phys. Rev. Lett. 107, 166802 (2011).

[61] C. Arnold, V. Loo, A. Lemaître, I. Sagnes, O. Krebs, P. Voisin, P. Senellart, and L. Lanco, Cavity-Enhanced Real-Time Monitoring of Single-Charge Jumps at the Microsecond Time Scale, Phys. Rev. X 4, 021004 (2014).

[62] S. Faez, S. J. van der Molen, and M. Orrit, Optical tracing of multiple charges in single-electron devices, Phys. Rev. B 90, 205405 (2014). 